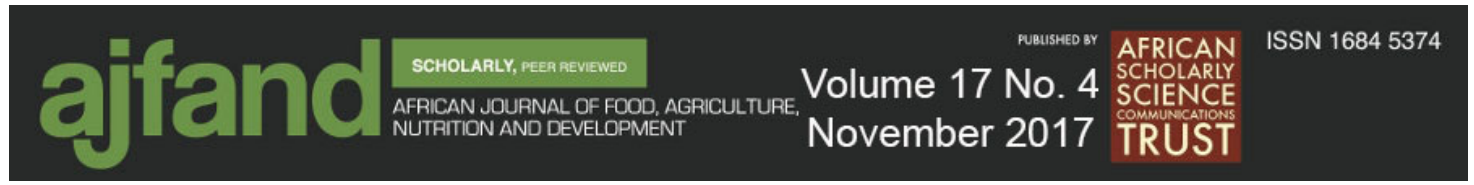

\title{
MODALITES DE CONSOMMATION ET VALEUR NUTRITIONNELLE DES LEGUMINEUSES ALIMENTAIRES AU BURKINA FASO
}

Hama-Ba $F^{1^{*}}$, Siedogo $M^{1-2}$, Ouedraogo $M^{3}$, Dao $A^{1}$, Dicko $H^{2}$, and B Diawara ${ }^{1}$

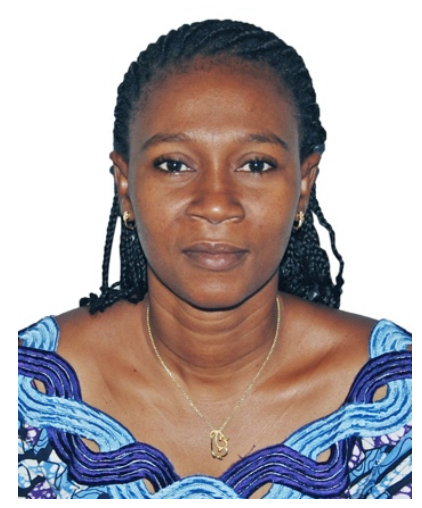

Fatoumata Hama-Ba

*Contact auteur de correspondance : hamafatou@yahoo.fr

${ }^{1}$ Institut de Recherche en Sciences Appliquées et Technologies/Département Technologie Alimentaire 03 BP 7047 Ouagadougou Burkina Faso

${ }^{2}$ Université de Ouagadougou. UFR SVT, Laboratoire BAEBIB

${ }^{3}$ Institut de l'Environnement et de Recherches Agricoles (INERA) 




RESUME

Au Burkina Faso les légumineuses constituent une source importante de nutriments pour les populations. La présente étude a eu pour objectif de déterminer la valeur nutritionnelle et la place des légumineuses dans le régime alimentaire des ménages à Ouagadougou, Kaya, Lebda et Nobéré. La méthodologie a consisté en une enquête de consommation alimentaire auprès de 325 ménages et à déterminer les teneurs en macronutriments et en minéraux fer, zinc et calcium de six (6) variétés de légumineuses. Il en résulte que les principales légumineuses consommées dans les ménages sont l'arachide (Arachis hypogea L.), le niébé (Vigna unguiculata L.), le voandzou (Vigna subterranea L.), le soja (Glycine max L.) et le zamnè (Acacia macrostachya R.). L'arachide, le niébé et le voandzou sont consommées plus 2 à 4 fois dans le mois par l'ensemble des ménages sous forme de mets au sein des ménages tandis que le soja et le zamnè sont consommés hors ménage. A l'exception du voandzou qui est consommé au déjeuner par 38,30\% des ménages à Ouagadougou, $60 \%$ à Kaya, $66,67 \%$ à Lebda et $43,18 \%$ à Nobéré, les autres légumineuses sont consommées à tout moment de la journée par plus de $80 \%$ des ménages enquêtés. Les légumineuses niébé, voandzou et zamnè sont consommées sous forme de ragout et de plats associés à des céréales respectivement par $99 \%, 93 \%$ et $76 \%$. L'arachide et le soja sont utilisés comme des ingrédients ou comme des collations. Les légumineuses ont des teneurs importantes en protéines, 35,76\%,31,04\%, 27,29\%, $22,55 \%$ et $20,38 \%$ respectivement pour le zamnè, le soja, l'arachide, le niébé et le voandzou. Les teneurs en lipides sont faibles et contribuent à moins de $7 \%$ à la valeur énergétique. Le niébé et le voandzou ont des teneurs en carbohydrates élevées, près de $60 \%$. Les teneurs en fer des légumineuses varient de $1,77 \mathrm{mg} / 100 \mathrm{~g}$ (voandzou) à 6,50 $\mathrm{mg} / 100 \mathrm{~g}$ (soja). Celles en zinc varient de $5,34 \mathrm{mg} / 100 \mathrm{~g}$ (zamnè) à 4,33 mg/100g (soja). Le soja et le zamnè ont d'importantes teneurs en calcium, respectivement $57,42 \mathrm{mg} / 100 \mathrm{~g}$ et $68,40 \mathrm{mg} / 100 \mathrm{~g}$. Du fait de leur valeur nutritionnelle intéressante en micronutriments ces deux légumineuses nécessitent une attention particulière pour l'enrichissement des produits locaux. La diversification des produits issus de ces légumineuses ainsi que celle des méthodes de consommation au sein des ménages permettront un meilleur profilage de leur bénéfice nutritionnel auprès des populations.

Mots clés : Légumineuses, importance, consommation, ménages, nutriments, urbain, rural, Burkina Faso 


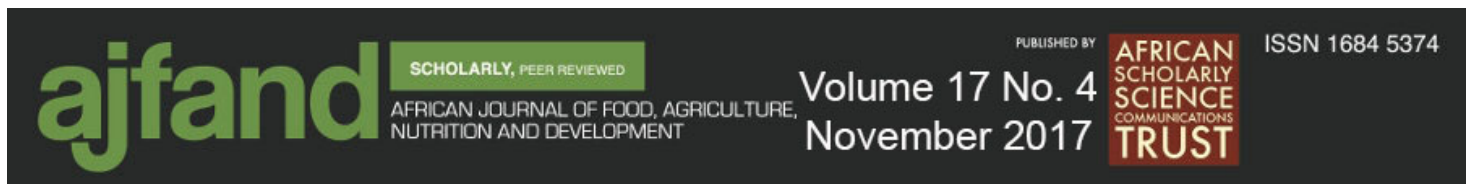

\begin{abstract}
In Burkina Faso legumes are an important source of nutrients for the populations. This study focused on the nutritional value and the place of legumes in the household diet. The study areas were Ouagadougou, Kaya, Lebda and Nobere. The methodology consisted of measurements of macronutrients and minerals iron, zinc contents and a survey from 325 households. The main legumes consumed in the households are groundnut (Arachis hypogea L.), cowpea (Vigna unguiculate L.), voandzou (Vigna subterranean L.), soybean (Glycine max L.) and zamnè (Acacia macrostachya R.). Groundnut, cowpea and voandzou are consumed more than 2 to 4 times in a month by all households in the form of meals within households while soybeans and zamnè are eaten outside the household. The other legumes are consumed at any time by more than $80 \%$ of the households surveyed, except for the voandzou, which is consumed at lunch by $38.30 \%$ of households in Ouagadougou, $60 \%$ in Kaya, $66.67 \%$ in Lebda and $43.18 \%$ in Nobéré. Cowpeas, voandou and zamnè legumes added to cereals are eaten as main dishes by respectively $99 \%, 93 \%$ and $76 \%$ of households. Groundnut and soybean are used as an ingredient or as a snack. Legumes have high contents of protein, $35.76 \%$, $31.04 \%, 27.29 \%, 22.55 \%$ and $20.38 \%$ respectively for zamne, soybean, groundnut, cowpea and voandzou. Lipid contents are low and contribute less than $7 \%$ to the energy value. Cowpeas and voandzou have high carbohydrate contents, nearly $60 \%$. The iron contents of legumes vary from $1.77 \mathrm{mg} / 100 \mathrm{~g}$ (voandzou) to $6.50 \mathrm{mg} / 100 \mathrm{~g}$ (soybean). Those in zinc range from $5.34 \mathrm{mg} / 100 \mathrm{~g}$ (zamnè) to $4.33 \mathrm{mg} / 100 \mathrm{~g}$ (soybean). Soybean and zamnè have high calcium contents, respectively $57.42 \mathrm{mg} / 100 \mathrm{~g}$ and $68.40 \mathrm{mg} / 100 \mathrm{~g}$. Soybean and zamnè require special attention because of the fortification of local product. of their interesting nutritional value in micronutrients. The diversification of products derived from these legumes and the methods of consumption within the households will allow a better profiling of the nutritional benefit of legumes for the populations.
\end{abstract}

Key words: legumes, importance, consumption, household, nutrients, urban, rural Burkina Faso 


\section{INTRODUCTION}

Au Burkina Faso, les légumineuses constituent une source importante de nutriments pour les populations, soit la $2^{\text {ème }}$ source protéique après les céréales avec un apport protéique de 18,6 g par jour contre 13,8 g pour les produits animaux. Leurs utilisations économiques font d'elles des cultures de choix pour répondre aux besoins de sécurité alimentaire dans les pays en développement [1]. Une grande diversité de légumineuses existe dans les différentes régions, avec près de 1004088 tonnes produites en 2016. Elles sont consommées sous forme de plats de base, de casse-croûte, de boisson. Seulement $58 \%$ de la production totale est consommée, soit une consommation annuelle par habitant de $31,1 \mathrm{~kg}$ contre $235 \mathrm{~kg}$ pour les céréales [2]. Ce sont des cultures de rente et une grande part est destinée à l'exportation, profitant très peu à la population. Les légumineuses ont des propriétés nutritionnelles et technologiques intéressantes [3, 4, 5]. En plus les légumineuses sont des sources de composés phytochimiques bénéfiques pour combattre les radicaux libres $[6,7]$. Le bénéfice nutritionnel de l'ajout des légumineuses aux céréales a fait l'objet de nombreux travaux $[8,9]$. A travers les résultats de la recherche de nombreuses méthodes de cuisson permettent d'améliorer la biodisponibilité des nutriments dans les légumineuses au profit des consommateurs [10]. Afin de mieux profiter des bienfaits des légumineuses, la $68^{\text {ième }}$ session de l'assemblée générale des Nations Unies a déclarée l'année 2016 « année internationale des légumineuses ». La présente étude s'intéresse aux modalités de consommation et à la valeur nutritionnelle des légumineuses en milieux rural et urbain du Burkina Faso.

Cette étude permettra de comprendre la place des légumineuses dans le régime alimentaire des populations au Burkina Faso afin de bien cibler les actions à entreprendre pour l'amélioration de l'état nutritionnel des populations.

\section{METHODOLOGIE}

\section{Zone de l'étude}

L'enquête de consommation des légumineuses a été réalisée dans deux milieux urbains et deux milieux ruraux. Les milieux urbains sont Ouagadougou et Kaya. Ouagadougou est la capitale du Burkina Faso et regroupe des populations diversifiées d'où son choix pour l'étude.

Les communes de Kaya, de Lebda et de Nobéré ont été choisies du fait de leur production importante en légumineuses. Kaya et Lebda font partir de la province de Sanmatenga dans la région du centre Nord. Kaya est la principale ville de la région et Lebda est une commune rurale située à $18 \mathrm{~km}$ de Kaya. Nobéré est une commune rurale, située dans la province de Zoundweogo au Centre Sud du pays.

\section{Echantillonnage}

L'enquête de consommation s'est intéressée à 325 ménages, soit 235 ménages à Ouagadougou, 30 ménages à Kaya, 30 ménages à Lebda, 30 ménages à Nobéré. La méthode statistique utilisée est la méthode aléatoire simple avec une marge d'erreur de $5 \%$ et un niveau de confiance de $95 \%$. Le dernier recensement général de la population a été considéré pour la population par localité [11]. 


\section{Questionnaire}

Le questionnaire a ciblé la personne qui s'occupe des repas dans le ménage. Il s'est intéressé au niveau de connaissance, aux modalités de consommation et à l'importance de la consommation des légumineuses dans le ménage.

\section{Analyses biochimiques \\ Collecte des échantillons}

Cinq légumineuses ont été collectées pour les analyses biochimiques pour l'étude (Tableau 1). Ils sont composés de six variétés de voandzou (Vigna subterranea L.), deux variétés de Niébé (Vigna unguiculata L.), la variété locale d'arachide (Arachis hypogea L.), la variété locale de Zamnè (Acacia macrostachya R.) et la variété locale de soja (Glycine max L.). Toutes les variétés ont été obtenues auprès des sélectionneurs de légumineuses de l'Institut de l'Environnement et de Recherches Agricoles (INERA).

\section{Préparation des échantillons}

A l'exception des graines d'arachide, les échantillons de légumineuses ont été traités de la même façon pour les analyses biochimiques. Les échantillons sont lavés séparément trois fois à l'eau potable et rincées à l'eau déminéralisée. Ils ont été ensuite séchés à l'étuve à $50^{\circ} \mathrm{C}$ pendant 48 heures avant d'être broyés à l'aide d'un broyeur IKA en inox. Les graines d'arachide sont également lavées et séchées puis dépelliculées avant d'être broyées. Les farines obtenues sont tamisées avec un tamis de 0,5 millimètre. Les résidus obtenus sont broyés de nouveau jusqu'à épuisement. Les farines fines sont gardées dans des pots stériles en polystyrène et conservées au réfrigérateur à $4^{\circ} \mathrm{C}$ pour les différentes analyses.

\section{Analyses biochimiques}

La teneur en eau des échantillons a été déterminée par pesée différentielle d'un échantillon de $5 \mathrm{~g}$ avant et après passage à l'étuve à $130^{\circ} \mathrm{C}$ pendant $2 \mathrm{~h}$ selon la norme française NF V 03-707, 2000 [12].

La teneur en protéines a été déterminée selon la méthode de Kjeldahl de la norme AFNOR NF V03-050 [13]. Le facteur de conversion considéré est de 6,25.

La teneur en lipides a été déterminée suivant la norme ISO-659 avec la méthode d'extraction au Soxhlet [14].

La teneur en carbohydrates a été obtenue par calcul [15] selon la formule : Teneur en carbohydrates $(\%)=100$ - [protéines $(\%)+$ lipides $(\%)+$ cendres $(\%)+$ eau $(\%)]$.

La teneur en fibre totale a été déterminée par la norme EN ISO 6865 [16].

Les teneurs en fer ont été déterminées par spectrométrie d'absorption atomique à flamme selon la méthode AOAC [17]. 
La valeur énergétique a été calculée en utilisant les coefficients d'Atwater et Benedict (1899) selon la formule suivante : Energie $(\mathrm{Kcal} / 100 \mathrm{~g})=\%$ glucides $\times 4(\mathrm{Kcal})+\%$ protéines $\times 4($ Kcal $)+\%$ lipides $\times 9($ Kcal $)[18]$.

\section{Analyses statistiques}

Les analyses nutritionnelles ont été réalisées en triple pour chaque échantillon et une moyenne a été calculée. Le logiciel SPSS Statistics 20 a été utilisé pour le traitement des données d'enquêtes et pour les tests de variance le niveau de confiance est de 95\%. Le tableur Excel a été utilisé pour les calculs des moyennes et des écarts types.

\section{RESULTATS ET DISCUSSION}

\section{Consommation des légumineuses}

Le tableau 2 présente le niveau de connaissance et de consommation des légumineuses dans les différentes localités. Sur 325 ménages, 97,85\% des ménages connaissent toutes les légumineuses et $70,15 \%$ en consomment au moins une. Les légumineuses sont bien connues et consommées dans toutes les localités de l'étude. Cependant un grand nombre de ménages principalement dans les centres urbains ne préparent pas les légumineuses soja, zamnè et voandzou. Le voandzou n'est préparé que par $42,13 \%$ des ménages à Ouagadougou. Près de $84,26 \%, 100 \%, 96,67 \%, 90 \%$ des ménages ne préparent pas le soja respectivement à Ouagadougou, Kaya, Lebda et Nobéré. Ces résultats indiquent une forte consommation de ces légumineuses individuellement hors du ménage. Ceci pourrait s'expliquer par la non adaptation ou la non maîtrise de la transformation du soja et du zamnè dans les ménages. Les graines de zamnè sont préparées de plus en plus au cours des grandes cérémonies coutumières et religieuses indiquant leur attachement culturel.

Les deux principales formes de consommation des légumineuses sont la sauce et les plats (Figure 1). Les légumineuses niébé, voandzou et zamnè sont consommées sous forme de ragout et de plats associés à des céréales respectivement par $99 \%, 93 \%$ et $76 \%$. Selon de nombreux travaux, l'addition des céréales aux légumineuses améliore la valeur nutritionnelle du plat. L'ajout des légumineuses a amélioré 2 à 3 fois la teneur en protéines des bouillies infantiles à base de céréales comparées aux bouillies importées comme le Cérélac [8]. Les légumineuses sont riches en acides aminés tryptophane et lysine qui sont déficients dans les céréales [20,21].

L'arachide et le soja sont principalement consommés sous forme de casse-croute. Les tourteaux, la pâte d'arachide, les brochettes de soja, le soumbala sont les principales formes de consommation. Des biscuits d'arachide sont consommés par $19 \%$ et $7 \%$ des ménages à Ouagadougou et à Lebda. Le soumbala est un ingrédient ajouté dans la préparation des sauces. Il est consommé en milieu rural, $63 \%$ des ménages à Nobéré et $37 \%$ à Kaya. Le lait de soja est consommé par 14\% des ménages à Ouagadougou mais très peu retrouvé dans les autres zones de l'étude. 

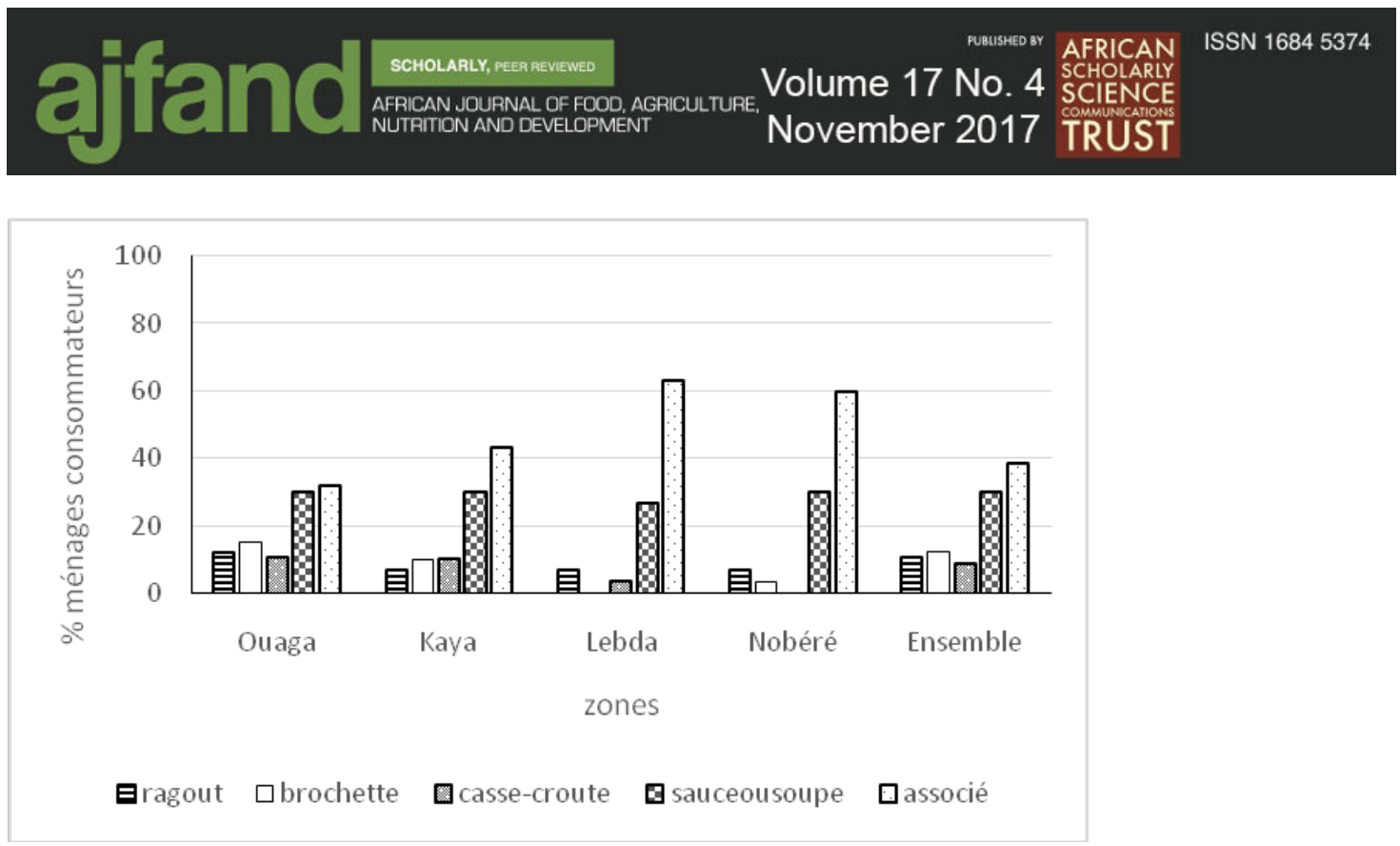

\section{Figure 1 : Forme de consommation des légumineuses}

La figure 2 présente les fréquences de consommation des légumineuses par localité. Il ressort une consommation fréquente des graines d'arachide et de niébé dans toutes les localités-plus de 4 fois dans le mois. La fréquence de consommation du soja, du voandzou et du zamnè se présente différemment dans les localités enquêtées. A Ouagadougou, $74,04 \%, 41,81 \%$ et $25,56 \%$ consomment plusieurs fois par mois le soja, le voandzou, et le zamnè. A Kaya, à Lebda et à Nobéré plus de 50\% des enquêtés consomment une fois par mois le soja et le zamnè. Le niébé ressort comme étant la légumineuse régulièrement consommée dans les ménages ruraux et urbains au Burkina Faso. La métaphore « le haricot est la viande du pauvre » traduit cette accessibilité des graines de niébé à des couches sociales modestes. Selon une étude réalisée par Life Sciences Research Office [19] en Amérique, les quantités de niébé consommées chez les hommes de plus de 20 ans pendant 3 jours augmentent inversement aux revenus.

Les légumineuses sont consommées à tout moment par les ménages. Seul le voandzou est consommé au déjeuner par 38,30\% des ménages à Ouagadougou, $60 \%$ à Kaya, $66,67 \%$ à Lebda et $43,18 \%$ à Nobéré. A Nobéré le niébé est consommé au petit déjeuner par près de 30,43\% et le voandzou au diner par 47,73\% des ménages. En effet le niébé et le voandzou sont riches en énergie d'où leur forte consommation au petit déjeuner et au déjeuner par certains consommateurs. 

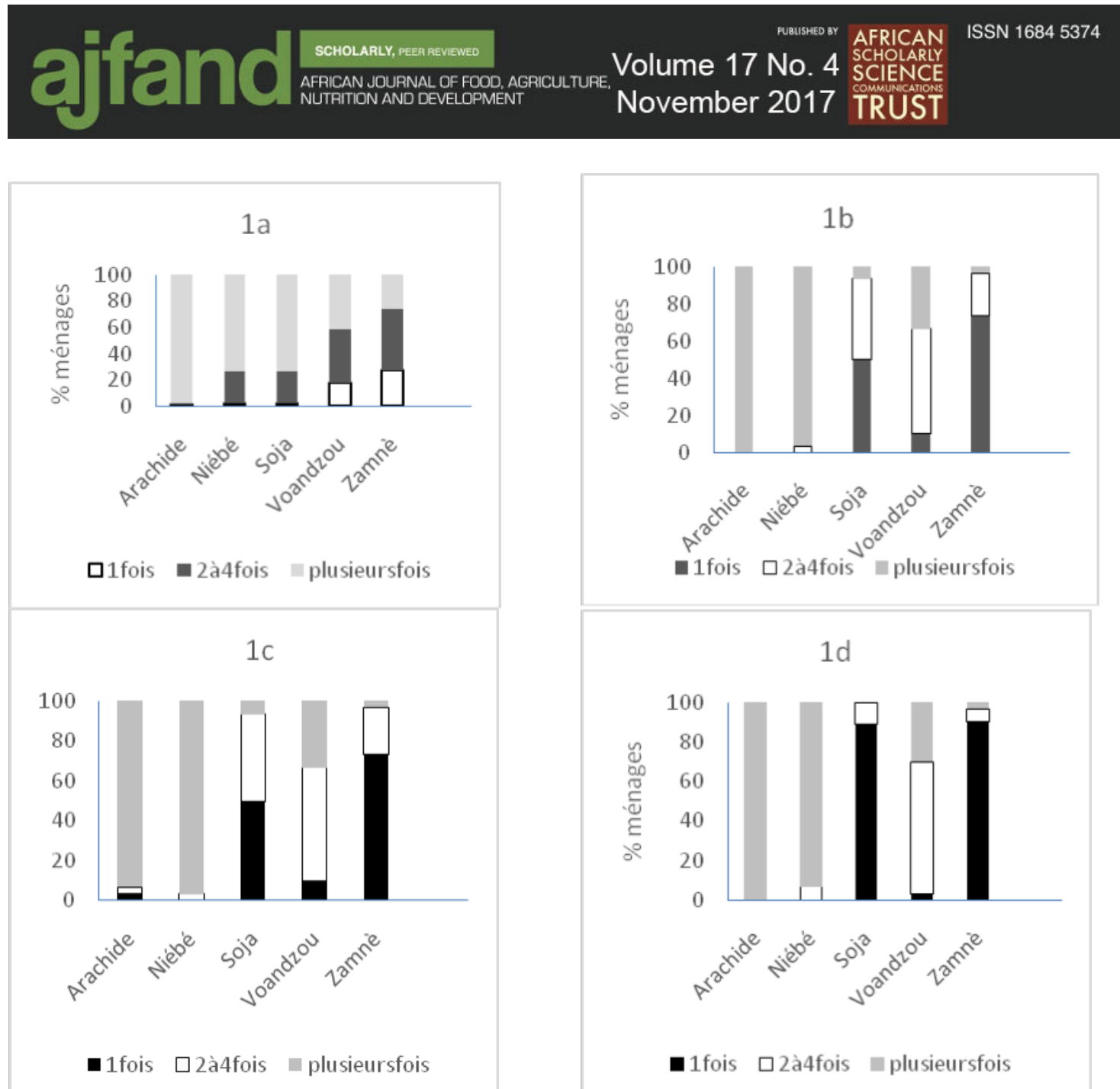

Figure 2 : Fréquence de consommation des légumineuses à Ouagadougou (1a), à Kaya (1b), à Nobéré (1c) et à Lebda (1d) dans le mois

La principale raison de non consommation ou de l'irrégularité de la consommation est l'inconfort dû au ballonnement après la consommation des légumineuses. Environ 20\% des ménages dont $18 \%$ provenant de Ouagadougou ont confirmé des ballonnements après la consommation des légumineuses. Cet inconfort réduit la consommation des légumineuses chez certaines personnes. Les oligosaccharides, 25 à $50 \%$ sont les principaux facteurs responsables de la flatulence [22]. En effet la muqueuse intestinale humaine ne dispose pas de $\alpha$-galactosidase pour cliver les oligosaccharides des molécules de stachyose, de raffinose. Ces molécules sont métabolisées dans le gros intestin par des bactéries formant des quantités importantes de dioxyde de carbone, d'hydrogène parfois de méthane. Une maîtrise des procédés de cuisson des légumineuses pourrait améliorer le niveau de consommation des légumineuses dans les ménages. Des études ont rapporté une amélioration de la digestibilité des graines de légumineuses par destruction ou inactivation de ces facteurs au cours de la cuisson [23, 24]. 


\section{Composition nutritionnelle des légumineuses}

Le tableau 5 présente les teneurs en macronutriments, en fibres et en micronutriments fer, zinc et calcium. Ces teneurs sont significativement différentes d'une légumineuse à une autre.

Les légumineuses sont d'excellentes sources de carbohydrates, de protéines et de fibres. Le niébé et le voandzou ont des teneurs en carbohydrates élevées, 1,5 fois supérieures à celles du soja et du Zamnè. Les résultats obtenus sont supérieurs à ceux trouvés sur des variétés de niébé consommées au Ghana et au Nigéria, avec des teneurs respectivement de $50,55 \%$ à $53,98 \%$ et $53.56 \%$ to $57.36 \%[1,26]$.

Les teneurs en protéines du zamnè, du soja, et de l'arachide sont respectivement 35,76 $\%, 31,04 \%$ et $27,29 \%$. Ces valeurs sont élevées comparées à celles des variétés de niébé et de voandzou qui sont respectivement de $22,55 \%$ et $20,38 \%$. En effet les légumineuses sont riches en protéines $[1,4,25]$. Des études ont rapporté des valeurs similaires sur des variétés de niébé au Ghana, 20,37\% à 25,28\% [4]. Des travaux réalisés sur des graines de zamnè au Burkina Faso ont rapporté des valeurs en protéines de 37,7\% [25]. Des valeurs plus faibles $10,44 \%$ à $13,06 \%$ ont été retrouvées par d'autres auteurs [25]. Ces différences pourraient être dues au type de sols, aux pratiques culturales, aux facteurs génétiques et environnementaux [1]. Au Burkina Faso l'espèce Acacia macrostachya est retrouvée dans les zones forestières et est accessible à la population rurale. D'où son importance dans l'amélioration nutritionnelle du régime alimentaire des populations rurales qui sont vulnérables aux carences nutritionnelles.

Les graines d'arachide ont la teneur en lipide la plus élevée, 46,72\%. Les plus faibles valeurs en lipides sont celles des Niébé $1,73 \%$ et du voandzou 6,74\%. L'arachide et le soja sont des légumineuses oléagineuses. Les lipides des légumineuses sont principalement constitués de triacylglycerol, d'acides gras libres, de phospholipides et de glycolipides.

L'arachide, le soja et le zamnè ont des teneurs en fibres élevées, respectivement de $30,77 \%, 15,61 \%$ et $15,68 \%$. Les teneurs en fibres des variétés de voandzou sont deux fois supérieures à celles des variétés de niébé. Des résultats similaires ont été rapportés sur des variétés de niébé consommés au Ghana, avec des teneurs de 1.56\% - 4.47\% [4]. Des teneurs en fibres insolubles de 74 à $78 \%$ ont été obtenues sur des variétés de soja ayant des teneurs similaires à celles de l'étude en Espagne [27]. L'IOM (Institute of Medecine Food and Nutrition Board) recommande la consommation d'aliments riches en fibres. En effet les fibres pourraient réduire les risques liés aux maladies cardiovasculaires et au cancer [28].

Les teneurs en fer des légumineuses varient de $1,77 \mathrm{mg} / 100 \mathrm{~g}$ (variétés voandzou) à 6,50 $\mathrm{mg} / 100 \mathrm{~g}$ (Soja). Les teneurs en zinc varient de $5,34 \mathrm{mg} / 100 \mathrm{~g}$ (zamnè) à 4,33 mg/100g (soja). Arachide et niébé ont des teneurs en zinc respectives de $3,86 \mathrm{mg} / 100 \mathrm{~g}$ et 4,03 $\mathrm{mg} / 100 \mathrm{~g}$ tandis que les variétés de voandzou ont une teneur de $2,46 \mathrm{mg} / 100 \mathrm{~g}$. Le soja et le zamnè ont d'importantes teneurs en calcium, respectivement $57,42 \mathrm{mg} / 100 \mathrm{~g}$ et 68,40 $\mathrm{mg} / 100 \mathrm{~g}$. Le soja et le zamnè sont des sources de micronutriments calcium, fer et zinc. La biodisponibilité du calcium dans les graines de soja est assez bonne et comparable à 


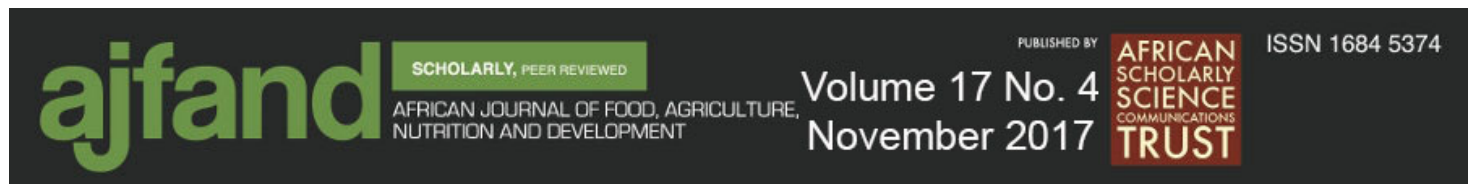

celle du calcium dans le lait animal malgré la forte teneur des graines de soja en phytates et en oxalate [29]. Les variétés de niébé et de soja sont riches en fer. Cependant la biodisponibilité du fer est faible dans les légumineuses mais celle du zinc est assez bonne $25 \%[30]$.

La faible biodisponibilité des micronutriments dans les légumineuses s'explique par la présence de facteurs inhibiteurs tels que les inhibiteurs de trypsine, les phytates, les oligosaccharides, les saponines [29].

\section{CONCLUSION}

Les légumineuses sont bien connues et consommées dans toutes les localités de l'étude. Les principales légumineuses utilisées pour les plats principaux dans les ménages sont l'arachide, le niébé et le voandzou. Les effets de flatulence et de ballonnement sont les principaux facteurs limitant la consommation. Les deux principales formes de consommation sont la sauce et les plats. Le soja est consommé sous forme de soumbala en milieu rural. Les légumineuses sont des sources importantes de protéines et de fer. Le soja et le zamnè du fait de leur valeur nutritionnelle intéressante en micronutriments nécessitent une attention particulière pour les formulations d'amélioration nutritionnelle. La diversification des produits issus de ces légumineuses, associée à des traitements pour réduire les facteurs de ballonnement permettront d'assurer un meilleur bénéfice nutritionnel des légumineuses auprès des populations.

\section{REMERCIEMENTS}

Nous adressons les remerciements à la Fondation Mcknight qui a financé cette étude. 


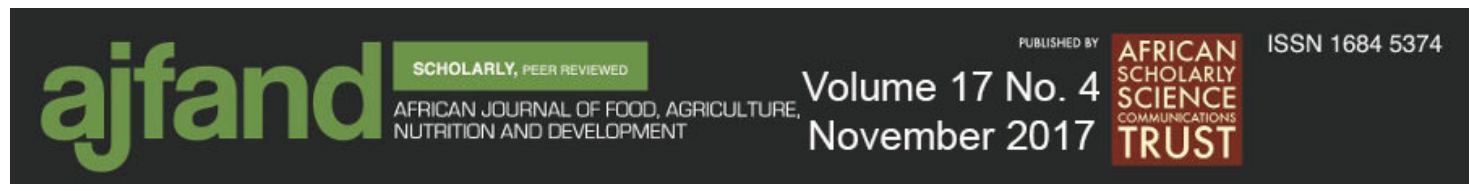

Tableau 1 : Nature des légumineuses de l'étude

\begin{tabular}{ll}
\hline Légumineuses & Variétés \\
\hline Arachide (Arachis hypogea) & Arachide locale \\
Soja (Glycine max) & Soja local Ouaga \\
Zamnè (Acacia macrostachya) & Zamnè local \\
Voandzou (Vigna subterranea) & Voandzou local Ouaga \\
& Voandzou Garga \\
& Voandzou KVS 075 \\
& Voandzou local Pobé \\
& Voandzou KVS 235 \\
& Voandzou KVS 246 \\
Niébé (Vigna unguilata) & Niébé KVx61-1 \\
& Niébé Niurzè \\
\hline
\end{tabular}




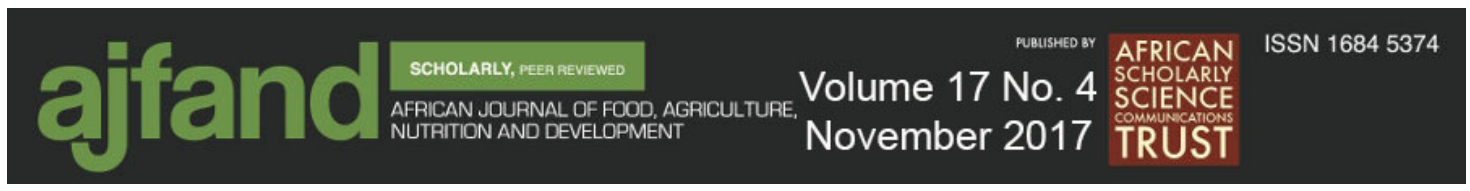

Tableau 2 : Niveau de connaissance des légumineuses dans les ménages par zone

\begin{tabular}{|c|c|c|c|c|}
\hline & Légumineuses & Connaissance & Consommation & $\begin{array}{l}\text { Non préparé } \\
\text { dans le ménage }\end{array}$ \\
\hline \multirow{7}{*}{ Ouagadougou } & Arachide & 100,00 & 100,00 & 0,43 \\
\hline & Niébé & 100,00 & 100,00 & 0,43 \\
\hline & Soja & 98,30 & 94,89 & 84,26 \\
\hline & Voandzou & 100,00 & 98,72 & 57,87 \\
\hline & Zamnè & 100,00 & 94,89 & 84,68 \\
\hline & Toutes & 98,30 & 91,06 & 0 \\
\hline & Au moins une & 100,00 & 100,00 & 97,01 \\
\hline \multirow{7}{*}{ Kaya } & Arachides & 100,00 & 100,00 & 0 \\
\hline & Niébé & 100,00 & 100,00 & 0 \\
\hline & Soja & 100,00 & 100,00 & 100 \\
\hline & Voandzou & 100,00 & 100,00 & 6,67 \\
\hline & Zamnè & 100,00 & 100,00 & 70 \\
\hline & Toutes & 100,00 & 100,00 & 0 \\
\hline & Au moins une & 100,00 & 100,00 & 30 \\
\hline \multirow{7}{*}{ Lebda } & Arachides & 100,00 & 100,00 & 0 \\
\hline & Niébé & 100,00 & 100,00 & 0 \\
\hline & Soja & 90,00 & 90,00 & 96,67 \\
\hline & Voandzou & 100,00 & 100,00 & 3,33 \\
\hline & Zamnè & 100,00 & 100,00 & 40 \\
\hline & Toutes & 90,00 & 90,00 & 0 \\
\hline & Au moins une & 100,00 & 100,00 & 30 \\
\hline \multirow{7}{*}{ Nobéré } & Arachides & 100,00 & 100,00 & 0 \\
\hline & Niébé & 100,00 & 100,00 & 0 \\
\hline & Soja & 100,00 & 100,00 & 90 \\
\hline & Voandzou & 100,00 & 100,00 & 6,67 \\
\hline & Zamnè & 100,00 & 100,00 & 96,67 \\
\hline & Toutes & 100,00 & 100,00 & 0 \\
\hline & Au moins une & 100,00 & 100,00 & 30 \\
\hline \multirow{2}{*}{ Total } & Toutes & 97,85 & 92,62 & 0 \\
\hline & Au moins une & 100,00 & 100,00 & 97,84 \\
\hline
\end{tabular}




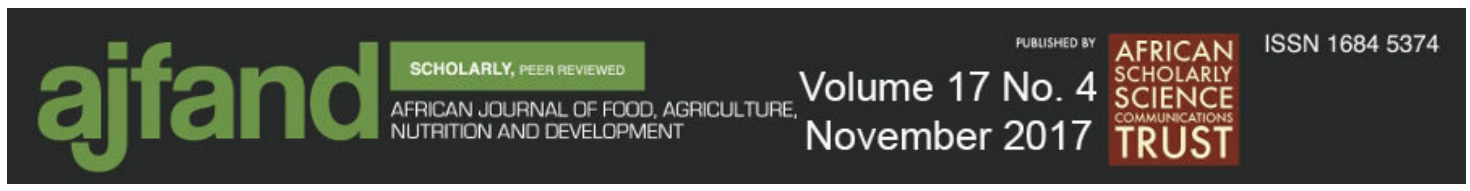

Tableau 3 : Moment de consommation des légumineuses par zone au cours de la journée

\begin{tabular}{clccccc}
\hline & & Arachide & Niébé & Soja & Voandzou & Zamnè \\
\hline \multirow{4}{*}{ Petit } & Ouagadougou & 0,00 & 2,13 & 2,20 & 2,13 & 2,13 \\
déjeuner & Kaya & 0,00 & 3,33 & 0,00 & 10,00 & 16,67 \\
& Lebda & 1,00 & 3,33 & 0,00 & 10,00 & 16,67 \\
& Nobéré & 3,03 & 30,43 & 0,00 & 2,27 & 0,00 \\
\hline \multirow{5}{*}{ Déjeuner } & Ouagadougou & 2,13 & 31,91 & 3,96 & 38,30 & 5,11 \\
& Kaya & 0,00 & 10,00 & 0,00 & 60,00 & 10,00 \\
& Lebda & 0,00 & 23,33 & 3,70 & 66,67 & 16,67 \\
& Nobéré & 12,12 & 28,26 & 9,38 & 43,18 & 0,00 \\
\hline \multirow{5}{*}{ Diner } & Ouagadougou & 1,70 & 17,87 & 6,17 & 9,36 & 1,70 \\
& Kaya & 0,00 & 0,00 & 0,00 & 6,67 & 0,00 \\
& Lebda & 0,00 & 6,67 & 0,00 & 10,00 & 3,33 \\
& Nobéré & 30,30 & 17,39 & 9,38 & 47,73 & 0,00 \\
\hline \multirow{2}{*}{ Peu } & Ouagadougou & 97,02 & 62,98 & 91,19 & 56,17 & 88,51 \\
& Kaya & 100,00 & 86,67 & 100,00 & 23,33 & 73,33 \\
& Lebda & 96,67 & 66,67 & 96,30 & 13,33 & 63,33 \\
& Nobéré & 54,55 & 23,91 & 81,25 & 6,82 & 100,00 \\
\hline
\end{tabular}




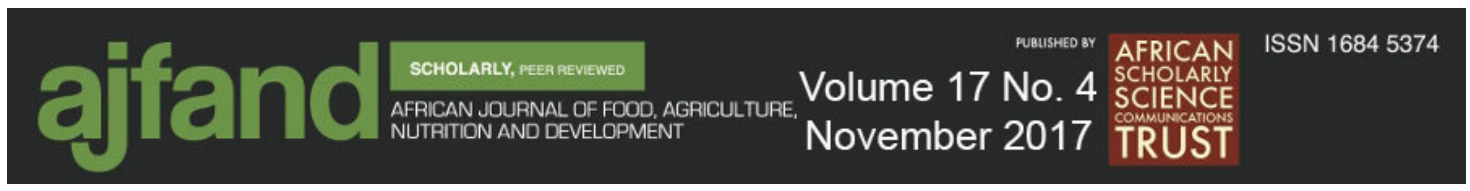

Tableau 4 : Consommation des mets par légumineuse dans les zones enquêtées

\begin{tabular}{llccccc}
\hline & & Ouagadougou & Kaya & Lebda & Nobéré & Ensemble \\
\hline \multirow{5}{*}{ Arachide } & Arachide & 100 & 100 & 100 & 100 & 100 \\
& Grillée/bouillie/crue & & & & & \\
& Pâte d'arachide & 100 & 100 & 100 & 100 & 100 \\
& Tourteaux & 89 & 0 & 0 & 90 & 91 \\
& Huile & 2 & 3 & 3 & 0 & 2 \\
& Biscuits/caramel & 19 & 0 & 7 & 0 & 15 \\
\hline \multirow{5}{*}{ Niébé } & Ragout & 98 & 93 & 83 & 100 & 96 \\
& Niébé-légumes & 98 & 100 & 100 & 100 & 98 \\
& Niébé-céréales & 100 & 100 & 97 & 100 & 99 \\
& Beignets & 99 & 100 & 100 & 100 & 99 \\
\hline \multirow{5}{*}{ Soja } & Brochettes & 94 & 97 & 93 & 97 & 95 \\
& Lait & 14 & 3 & 0 & 0 & 10 \\
& Soumbala & 2 & 37 & 23 & 63 & 13 \\
\hline \multirow{5}{*}{ Voandzou } & Ragout/tô/gnon & 89 & 97 & 83 & 100 & 90 \\
& Voandzou-céréales & 91 & 97 & 100 & 97 & 93 \\
& Gâteau/crêpes/grillé & 7 & 0 & 0 & 0 & 0 \\
\hline \multirow{5}{*}{ Zamnè } & Ragout & 88 & 70 & 27 & 90 & 81 \\
& Associé & 75 & 53 & 83 & 97 & 76 \\
& Sauce & 31 & 0 & 0 & 17 & 24 \\
\hline
\end{tabular}




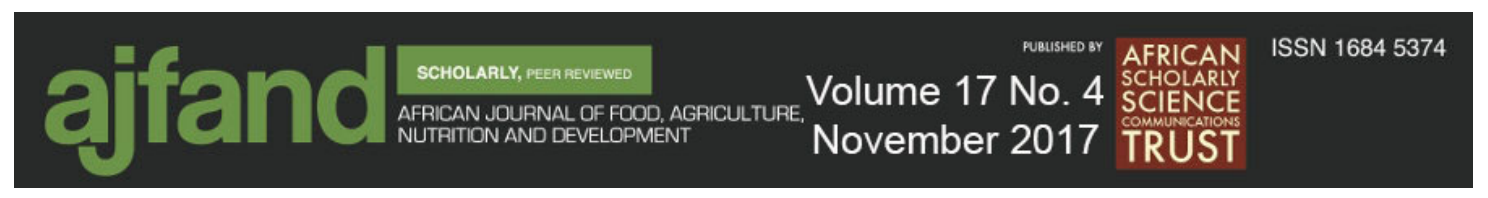

Tableau 5 : Composition en macronutriments et minéraux des légumineuses

\begin{tabular}{|c|c|c|c|c|c|c|c|c|c|c|c|c|c|c|c|c|c|c|c|c|c|}
\hline \multirow[b]{2}{*}{ Arachide } & \multicolumn{3}{|c|}{$\begin{array}{c}\text { Carbohydrates } \\
(\%)\end{array}$} & \multicolumn{3}{|c|}{ Protéines $(\%)$} & \multicolumn{3}{|c|}{ Lipides (\%) } & \multicolumn{3}{|c|}{ Fibres $(\%)$} & \multicolumn{3}{|c|}{$\begin{array}{l}\text { Calcium } \\
(\mathrm{mg} / \mathbf{1 0 0 g})\end{array}$} & \multicolumn{3}{|c|}{$\begin{array}{c}\text { Fer } \\
(\mathrm{mg} / 100 \mathrm{~g})\end{array}$} & \multicolumn{3}{|c|}{$\begin{array}{c}\text { Zinc } \\
(\mathrm{mg} / \mathbf{1 0 0 g})\end{array}$} \\
\hline & 19,30 & \pm & 0,10 & 27,29 & \pm & 0,03 & 46,72 & \pm & 0,03 & 30,77 & \pm & 0,01 & 5,73 & \pm & 0,60 & 2,24 & \pm & 0,13 & 4,03 & \pm & 0,07 \\
\hline Niébé KVX61-1 & 64,08 & + & 0,03 & 21,90 & \pm & 0,02 & 1,43 & + & 0,02 & 3,04 & \pm & 0,06 & 18,21 & \pm & 1,24 & 3,66 & \pm & 0,15 & 3,96 & \pm & 0,04 \\
\hline Niébé Niizwé & 63,30 & + & 0,04 & 23,20 & \pm & 0,03 & 2,03 & + & 0,00 & 3,28 & \pm & 0,24 & 14,99 & \pm & 0,81 & 3,94 & \pm & 0,59 & 3,82 & \pm & 0,11 \\
\hline Soja & 41,93 & \pm & 0,02 & 31,04 & \pm & 0,00 & 18,73 & \pm & 0,01 & 15,61 & \pm & 0,12 & 57,42 & \pm & 0,37 & 6,50 & \pm & 0,57 & 4,33 & \pm & 0,03 \\
\hline Voandzou Garga & 61,61 & + & 0,02 & 19,70 & \pm & 0,03 & 6,43 & + & 0,08 & 7,26 & \pm & 0,20 & 12,26 & \pm & 0,07 & 1,48 & \pm & 0,01 & 2,44 & \pm & 0,01 \\
\hline Voandzou KVS 075 & 61,51 & \pm & 0,08 & 20,37 & \pm & 0,04 & 6,81 & $\perp$ & 0,00 & 8,38 & \pm & 0,05 & 11,48 & \pm & 0,23 & 1,47 & \pm & 0,34 & 2,26 & \pm & 0,01 \\
\hline Voandzou KVS 235 & 60,84 & \pm & 0,04 & 20,99 & \pm & 0,01 & 6,58 & 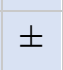 & 0,06 & 8,25 & \pm & 0,35 & 12,19 & \pm & 0,29 & 1,73 & \pm & 0,14 & 2,27 & \pm & 0,01 \\
\hline Voandzou KVS 246 & 62,12 & $I$ & 0,05 & 19,41 & \pm & 0,02 & 7,35 & + & 0,05 & 7,48 & \pm & 0,03 & 13,15 & \pm & 2,96 & 1,39 & \pm & 0,29 & 2,37 & \pm & 0,00 \\
\hline Voandzou local & 59,39 & + & 0,05 & 21,64 & \pm & 0,01 & 6,78 & \pm & 0,08 & 6,84 & \pm & 0,13 & 10,77 & \pm & 1,37 & 2,60 & \pm & 0,72 & 2,73 & \pm & 0,06 \\
\hline Voandzou Pobé & 60,51 & \pm & 0,08 & 20,21 & \pm & 0,00 & 6,53 & $\perp$ & 0,07 & 6,84 & \pm & 0,13 & 10,71 & \pm & 0,05 & 1,95 & \pm & 0,14 & 2,69 & \pm & 0,04 \\
\hline Zamnè & 40,87 & \pm & 0,12 & 35,76 & \pm & 0,06 & 9,04 & \pm & 0,01 & 15,68 & \pm & 0,12 & 68,40 & \pm & 0,37 & 4,77 & \pm & 0,80 & 5,34 & \pm & 0,06 \\
\hline
\end{tabular}




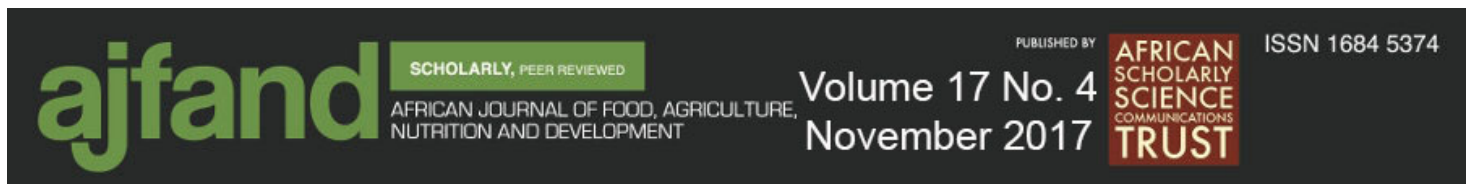

\section{REFERENCES}

1. Appiah F, Asibuo JY and P Kumah Physical and functional properties of bean flours of three cowpea (Vigna unguiculata L. walp) varieties in Ghana. African Journal of Food Science, 2011; 5(2): 100-104.

2. Résultats définitifs de la campagne agricole et de la situation alimentaire et nutritionnelle 2015/2016. Ministère de l'Agriculture et de la Sécurité Alimentaire / Direction Générale des Etudes et Statistiques Sectorielles (DGESS), Ouagadougou, Burkina Faso, 2016.

3. Savadogo A, Ilboudo AJ and AS Traoré Nutritional Potentials of Acacia Macrostachya (Reichend) ex Dc Seeds of Burkina Faso: Determination of Chemical Composition and Functional Properties. Journal of Applied Sciences Research, 2011; 7(7): 1057-1062.

4. Aaron AT, Robert A, Adukpo GE, Diabor E and AA Kingsley Assessment of functional properties and Nutritional composition of some cowpea (Vigna unguiculata L.) genotypes in Ghana ARPN. Journal of Agricultural and Biological Science, 2013; 8(6).

5. Hama-Ba F, Silga P and B Diawara Evaluation de la qualité et de l'acceptabilité de couscous à base de trois formulations de farines composites enrichies au soja (Glycine max) et au moringa (Moringa oleifera). Int. J. Biol. Chem. Sci., 2016 ; 10(6) : 2497-2510.

6. Tiwari $\mathbf{U}$ and $\mathbf{E}$ Cummins Factors influencing levels of phytochemicals in selected fruit and vegetables during pre- and post-harvest food processing operations. Food Res. Int., 2013; 50(2): 497-506.

doi:10.1016/j.foodres.2011.09.007.

7. Curran $\mathbf{J}$ The nutritional value and health bene fits of pulses in relation to obesity, diabetes, heart disease and cancer. Br. J. Nutr. 2012; 108(S1): S1-S2.

8. Adriana FDT and AG Crosby A review of the impact of preparation and cooking on the nutritional quality of vegetables and legumes. International Journal of Gastronomy and Food Science, 2016; 3: 2-11.

9. Solomon Mariam Nutritive value of three potential complementary foods based on cereals and legumes. African Journal of Food and Nutritional Sciences, 2005; 5(2): $1-14$.

10. Kayodé APP, Akogou FGU, Amoussa Hounkpatin W and DJ Hounhouigan Effets des procédés de transformation sur la valeur nutritionnelle des formulations de bouillies de complément à base de sorgho Int. J. Biol. Chem. Sci., $2012 ; \mathbf{6}(\mathbf{5})$ : 2192-2201. 
11. Recensement Général de la Population et de l'Habitation Institut National de Statistiques et de la Démographie. Ministère de l'Economie et des Finances. Burkina Faso, 2006.

12. Association Française de Normalisation (AFNOR). Détermination de la teneur en eau, méthode pratique. Céréales, Légumineuses, Produits Dérivés NF V 03$707,2000$.

13. Association Française de Normalisation (AFNOR). Directives générales pour le dosage de l'azote avec minéralisation selon la méthode de Kjedahl. Produits Agricoles Alimentaires, NF V 03-050, 1970.

14. ISO (International Standardization Organization) Détermination de la teneur en matière grasse selon la méthode d'extraction par Soxhlet, ISO 659, 1998.

15. Egan H, Kirk RS and PR Sawyer Chemical Analyses of Food (8th edition). Churchill. Livingstone: London-UK, 591p, 1981.

16. ISO (International Standardization Organization) Détermination de la teneur en Fibres selon la norme EN ISO 6865, 2000.

17. AOAC (Association of Official Analytical Chemists) Arlington, Virginia, USA, 2012.

18. Atwater WO and FG Benedict Experiments on the metabolism of matter and energy in the human body. US Department of Agriculture. Washington, D.C., Bulletin 69, 112p, 1899.

19. Life Sciences Research Office Federation of American Societies for Experimental Biology. Third report on nutrition monitoring in the United States, Vol 1. Washington, DC: US Government Printing Office, 1995.

20. Giami SY Compositional and nutritional properties of selected newly developed lines of Cowpea (Vigna unguiculata L. Walp). Journal of Food Composition and Analysis, 2005; 18: 665-673.

21. Duranti M Grain legume proteins and nutraceutical properties. Fitoterapia, 2006; 77: 67-82.

22. Carlsson GN, Karlsson $\mathbf{H}$ and AS Sandberg Determination of oligosaccharides in foods, diets, and intestinal contents by high temperature gas chromatography and gas chromatography/mass spectrometry. J Agric Food Chem., 1992; 40:2404-12.

23. Ramírez-Cárdenas L, Leonel AJ, Costa NMB and FP Reis Zinc bioavailability in different beans as affected by cultivar type and cooking conditions. Food Res. Int. 2010; 43(2): 573-581 doi: 10.1016/j.foodres. 2009.07.023. 
24. Wang N, Hatcher DW, Tyler RT, Toews R and EJ Gawalko Effect of cooking on the composition of beans (Phaseolus vulgaris L.) and chickpeas (Cicer arietinum L.). Food Res. Int., 2010a; 43 (2): 589-594. doi: 10.1016/j.foodres. 2009.07.012.

25. Ouattara S Utilisation des graines de Acacia macrostachya Reichend. ex De. comme source de protéines dans l'alimentation des poulets de chair. Diplôme d'Études Approfondies (DEA) en Gestion Intégrée des Ressources Naturelles, Spécialité: Production animale, Option: Nutrition et Alimentation Animale, 2008.

26. Chinma CE, Alomede CI and GI Emelife Physicochemical and Functional Properties of Some Nigerian Cowpea Varieties. Pak. J. Nutr. 2008; 7(1): 186190.

27. Redondo-Cuenca A, Rodríguez-Sevilla MD and I Mateos-Aparicio Chemical composition and dietary fiber of yellow and green commercial soybeans (Glycine max). Food Chemistry, 2007; 101(3): 1216-1222.

28. Institute of Medicine of the National Academies-IOM, Food and nutrition board. Dietary Reference Intakes: Energy, Carbohydrates, Fiber, Fat, Fatty acids, Cholesterol, Protein and Amino Acids. The National Academies Press, Washington, DC, 2005.

29. Vila-Donat P, Caprioli G, Conti P, Maggi F, Ricciutelli M and E Torregiani Rapid quantification of soyasaponins I and bg in Italian lentils by high performance liquid chromatography (HPLC)-tandem mass spectrometry (MS/ MS). Food Analytical Methods, 2014; 7: 1024-1031.

30. Sandström B, Almgren A, Kivistö B and A Cederblad Effect of protein level and protein source on zinc absorption in humans. Journal of Nutrition, 1989; 119: $48-53$. 